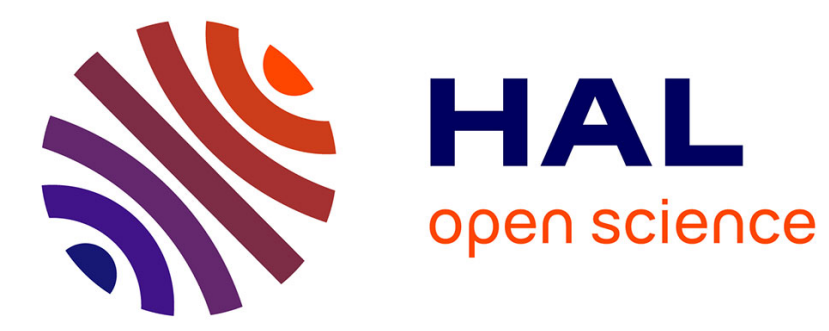

\title{
One-step and eco-friendly modification of cellulose membranes by polymer grafting
}

\author{
Julie Credou, Rita Faddoul, Thomas Berthelot
}

\section{To cite this version:}

Julie Credou, Rita Faddoul, Thomas Berthelot. One-step and eco-friendly modification of cellulose membranes by polymer grafting. RSC Advances, 2014, 4, pp.60959-60969. 10.1039/C4RA11219A . hal-01156572

\section{HAL Id: hal-01156572 \\ https://hal.science/hal-01156572}

Submitted on 17 Nov 2015

HAL is a multi-disciplinary open access archive for the deposit and dissemination of scientific research documents, whether they are published or not. The documents may come from teaching and research institutions in France or abroad, or from public or private research centers.
L'archive ouverte pluridisciplinaire HAL, est destinée au dépôt et à la diffusion de documents scientifiques de niveau recherche, publiés ou non, émanant des établissements d'enseignement et de recherche français ou étrangers, des laboratoires publics ou privés. 


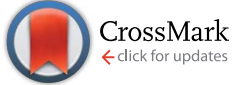

Cite this: RSC Adv., 2014, 4, 60959

\title{
One-step and eco-friendly modification of cellulose membranes by polymer grafting
}

\author{
Julie Credou, Rita Faddoul and Thomas Berthelot*
}

The increasing environmental awareness has stimulated the use of bio-based materials and processes. As an affordable and sustainable biopolymer, cellulose is an ideal engineering material. Beyond paper, cellulose finds applications in many areas such as composites, electronics and drug delivery. To fulfil these new functions, cellulose needs to acquire new properties, which is commonly done by graft polymerization of acrylic compounds. While cellulose modification is usually performed through complex and expensive procedures, the diazonium-based polymer grafting procedure presented here was performed in water, at room temperature, in a short single step. Cellulose sheets have been successfully grafted with several acrylic polymers, first globally through a dipping procedure and then locally by inkjet printing. The process developed herein is simple, eco-friendly and mostly time and cost-saving. More generally, it is a powerful tool for easy, robust and patterned graft copolymerization of cellulose sheets with various acrylic monomers and even bio-based monomers.

Received 25th September 2014 Accepted 4th November 2014

DOI: 10.1039/c4ra11219a

www.rsc.org/advances

Beyond paper and cardboard, cellulose thus finds applica-

\section{Introduction}

The increasing environmental awareness and the growing will for sustainable technologic development have stimulated the use of biosourced materials and the development of bio-based processes worldwide. Besides, the current economic global issues have incited a search for cost-saving approaches. ${ }^{\mathbf{1 , 2}}$ As the main material of plant cell walls, cellulose is the largest form of worldwide biomass (about $1.5 \times 10^{12}$ tons per year). ${ }^{3}$ This biopolymer is therefore the most abundant organic raw material on earth. ${ }^{1}$ In addition to its large bioavailability, good biodegradability and biocompatibility, its high functionality and relatively high chain stiffness make cellulose an extremely interesting polymer. ${ }^{4-8}$ Moreover, it is insoluble in most usual organic solvents and therefore is considered an ideal structural engineering material. ${ }^{7}$ It swells but does not dissolve in water, hence enabling aqueous fluids and their contained components to penetrate within the fibers matrix and to wick by capillarity with no need for any external power source. In addition, cellulose sheets are available in a broad range of thicknesses and well-defined pore sizes, easy to store and handle, and lastly safely disposable. ${ }^{9-11}$ Furthermore, the recent impetus given to paper-based microfluidics by American, Canadian and Finnish research teams ${ }^{\mathbf{1 2 - 1 4}}$ has resulted in the development of new paper-based devices for diagnostics, microfluidics, and electronics. ${ }^{7,15}$

CEA Saclay, IRAMIS, NIMBE, LICSEN (Laboratory of Innovation in Surface Chemistry and Nanosciences), F-91191 Gif sur Yvette, France. E-mail: julie.credou@cea.fr; rita. faddoul@cea.fr; thomas.berthelot@cea.fr; Fax:+33 169084044; Tel: +33 169086588 tions in many diverse areas such as composite materials, textiles, drug delivery systems and personal care products. ${ }^{2}$ In order to increase its functionality and the scope of its use, modifications of cellulose biofibers are required. By introducing lots of new functional moieties in one reaction, graft polymerization enables to rapidly alter the physical and chemical properties of cellulose and increase its functionality without destroying its many appealing intrinsic properties. ${ }^{2}$ Many properties can be improved or added to cellulose by polymer grafting including hydrophobicity, oil repellency, antimicrobial activity, heat resistance and electrical properties, dimension stability, resistance to abrasion and wear, wrinkle recovery. As an example, polyacrylic acid may be grafted to cellulose in order to complex metal cations. If silver cation is chelated, its reduction will result in paper decorated with silver nanoparticles which will endow the membrane with antibacterial activity. All these potential additional features allow cellulose to be used for advanced material applications. ${ }^{4}$

Cellulose graft copolymerization is usually performed by free radical polymerization of vinylic compounds in heterogeneous conditions, i.e. on a solid cellulose substrate with the monomer being in solution. Grafted side chains are initiated by radical formation on the cellulose backbone. This radical may originate from the homolytic bond cleavage within the glucose unit caused by high-energy irradiation for example, from the decomposition of a functional group such as peroxide, or from a radical transfer reaction initiated by a radical formed outside the cellulose backbone during a redox reaction., ${ }^{2,16}$ There are three kinds of approaches to covalent attachment of polymers to surfaces: (i) the "grafting-to" method, where a polymer is 
coupled with the functional moieties from cellulose backbone, (ii) the "grafting-from" method, where copolymer chains grow from initiating sites on the cellulose backbone, and (iii) the "grafting-through" method, where the cellulose bares a polymerizable group, and hence acts as a macromonomer with which a smaller monomer copolymerizes. Among these three methodologies, the "grafting-from" approach is the most commonly used procedure. ${ }^{2,4}$ Consequently, the work presented here focuses on the widely employed "grafting-from" free radical graft copolymerization of cellulose with acrylic compounds.

Many studies have reported cellulose graft copolymerization with acrylic compounds. ${ }^{17-23}$ Cellulose modifications have usually been performed under harsh conditions, in organic solvent or with highly toxic compounds so far. ${ }^{17-19,21}$ Besides graft polymerization most often implements long-lasting, complex and/or expensive procedures such as ATRP (Atom Transfer Radical Polymerization), ${ }^{17}$ RAFT (Reversible AdditionFragmentation chain Transfer polymerization) ${ }^{\mathbf{2 0}}$ and gamma irradiation initiation step. ${ }^{18,20,21}$ To the best of our knowledge, no cellulose modification has been done in a rather short onestep reaction and under soft conditions, i.e. in water and at room temperature.

Herein, a simple, fast, low-cost and eco-friendly way for graft copolymerization of cellulose sheets under soft and biocompatible conditions is presented. The cellulose modifications were performed in a single step, in water and at room temperature, in one hour or less. The cellulose modification pathway consisted in an aryldiazonium-based polymerization of acrylic monomers (GraftFast ${ }^{\mathrm{TM}}$ ). ${ }^{\mathbf{2 4 - 2 6}}$ Two different dispensing methods were employed to impregnated cellulose sheets with copolymerization reaction mixture. Firstly, dipping was performed. Though ecologically friendly, the process produced lots of matter wastage and was therefore not economically friendly. Thus, inkjet printing was further implemented to reduce this wastage by localizing the polymerization mixture onto specific areas of the substrate. Moreover, this versatile dispensing method is considered as a competitive method for patterning flexible or rigid substrates. It is a fast, cost-effective, additive, biocompatible and environmentally friendly method for depositing thin or thick films $(0.8-20.0 \mu \mathrm{m})$ according to complex patterns. ${ }^{27}$ Cellulose paper sheets have been successfully copolymerized (or printed and copolymerized) without damaging their intrinsic properties or even their visual aspect. Several acrylic monomers were compared. Furthermore, the inkjet printing process previously described ${ }^{28}$ was proved to be an efficient method allowing the patterning of cellulose tapes with grafted polymers.

\section{Experimental}

\subsection{Reagents and reaction materials}

4-nitrobenzenediazonium tetrafluoroborate, acrylic acid (AA), 2hydroxyethyl methacrylate (HEMA), methyl methacrylate (MMA), poly(ethylene glycol) dimethacrylate (PEGDMA), Lascorbic acid (vitamin C), tris(bipyridine)ruthenium(II) chloride $\left(\left[\mathrm{Ru}(\mathrm{bpy})_{3}\right] \mathrm{Cl}_{2}\right)$ and polyacrylic acid $\left(M_{\mathrm{w}}=130000 \mathrm{~g} \mathrm{~mol}^{-1}\right)$ were purchased from Sigma-Aldrich (St Louis, MO, USA) and used as received. Water used in all experiments was purified by the Milli-Q system (Millipore, Brussels, Belgium). CF1 cellulose paper was from Whatman (Maidstone, Kent, UK). In the first set of experiments, substrates were dipped into polymerization solutions. In the second one, the polymerization solution was printed onto substrates using a laboratory piezoelectric dropon-demand inkjet printer Dimatix Materials Printer DMP-2831 (Fujifilm, Santa Clara, CA, USA) with 10 pL nominal drop volume cartridge. Irradiations were carried at $453 \mathrm{~nm}$ at room temperature with a Golden Dragon Plus, deep blue LED (OSRAM Opto Semiconductors, Sunnyvale, CA, USA).

\subsection{Characterization materials}

Infrared (IR) spectra of the various substrates were recorded on a Vertex 70 FT-IR spectrometer (Bruker, Billerica, MA, USA) controlled by OPUS software (Bruker, Billerica, MA, USA) and fitted with MIRacle ${ }^{\mathrm{TM}}$ ATR (Attenuated Total Reflectance) sampling accessory (PIKE Technologies, Madison, WI, USA). The ATR crystal type was single reflection diamond/ZnSe crystal plate. The FT-IR detector was MCT working at liquid nitrogen temperature. Acquisitions were obtained at $2 \mathrm{~cm}^{-1}$ resolution after 256 scans.

Microstructure and surface morphology of samples were examined by a JSM-5510LV (JEOL, Tokyo, Japan) scanning electron microscope (SEM) after gold coating (K575X Turbo Sputter Coater (Quorum Technologies Ltd, Ashford, Kent, UK), working at $15 \mathrm{~mA}$ for 20 seconds). The images were acquired at various magnifications ranging from $100 \times$ to $3000 \times$. The acceleration voltage and working distance were $4 \mathrm{kV}$ and 17 $\mathrm{mm}$, respectively. Images were acquired applying the secondary electron detector.

Surface roughness, $R_{\mathrm{a}}$, of pristine and copolymerized cellulose substrates was measured with an AlphaStep ${ }^{\circledR}$ D-120 Stylus Profiler (KLA-Tencor, Milpitas, CA, USA). Measurements were performed along a line of $1 \mathrm{~mm}$ long, with a stylus force of $1 \mathrm{mg}$ and at a speed of $0.05 \mathrm{~mm} \mathrm{~s}^{-1}$. The same profiler was used to measure printed polyacrylic acid films thickness and roughness.

Ink viscosity was measured with a MCR 102 Rheometer (Anton Paar, Ashland, VA, USA). Cone-plane geometry was used at a shear rate varying from 100 to $5000 \mathrm{~s}^{-1}$ and at a $24{ }^{\circ} \mathrm{C}$ temperature. Gap distance was equal to $101 \mu \mathrm{m}$. Geometry diameter and angle were equal to $5 \mathrm{~cm}$ and $1^{\circ}$, respectively.

$\mathrm{X}$-ray powder diffraction (XRPD) patterns were measured using a high-throughput Bruker D8 Advance diffractometer (Bruker, Billerica, MA, USA) working on transmission mode and equipped with a focusing Göbel mirror producing $\mathrm{CuK} \alpha$ radiation $(\lambda=1.5418 \AA)$ and a LYNXEYE detector. Data were collected at room temperature (RT), in the $2 \theta$ range $3-60^{\circ}$, with a $0.02^{\circ}$ step width.

\subsection{Cellulose graft copolymerization}

2.3.1. Dipping procedure. Cellulose modification was performed in water, at open air and room temperature. The $2 \mathrm{~mL}$ aqueous reaction mixture contained $0.10 \mathrm{mmol}$ of diazonium 
salt (23.69 mg; 1.0 eq.), $2 \mathrm{mmol}$ of monomer (20 eq.) and 0.01 mmol of L-ascorbic acid (1.76 mg; 0.1 eq.). Components were first separately dissolved in water and then mixed under stirring in the following order: (i) monomer, (ii) diazonium salt, and (iii) L-ascorbic acid. A CF1 paper sheet $\left(4 \mathrm{~cm}^{2}\right)$ was dipped into this freshly prepared mixture and left to incubate for one hour in a plastic box. The membrane obtained was rinsed and submitted to ultrasonic treatment in order to discard any ungrafted matter. A first wash was made with water, a second with ethanol. It was finally dried for 15 minutes at $60{ }^{\circ} \mathrm{C}$ in an air oven. Several acrylic polymers were thus grafted to cellulose paper. The corresponding monomers were acrylic acid (AA), 2-hydroxyethyl methacrylate (HEMA), methyl methacrylate (MMA) and poly(ethylene glycol) dimethacrylate (PEGDMA). All the so functionalized papers were then analyzed using infrared spectroscopy in order to point out the carbonyl moieties brought by the polymerization. Their microstructure was pictured by SEM imaging. Their surface roughness was measured with a profiler.

\subsubsection{Printing procedure}

2.3.2.1. Ink formulation. Ink formulation was inspired from our previous work for flexible electronic interconnects. ${ }^{28}$ First of all, an aqueous mixture of acrylic acid (AA) monomer and polyacrylic acid (PAA) (13 wt\% of the $14.6 \mathrm{M}$ commercial stock solution and $2.5 \mathrm{wt} \%$ of a $1 \mathrm{wt} \%$ aqueous stock solution, respectively) was prepared. Afterwards, $0.8 \mathrm{wt} \%$ of solid 4nitrobenzenediazonium tetrafluoroborate (NBD) was added to the previous solution. Finally, tris(bipyridine)ruthenium(II) chloride $\left(\left[\mathrm{Ru}(\mathrm{bpy})_{3}\right] \mathrm{Cl}_{2}\right)(1.3 \mathrm{wt} \%$ of a $0.02 \mathrm{M}$ aqueous stock solution) was added to the ink. Hence, the final ink composition was: $2 \mathrm{M}$ of AA, traces of PAA, $0.03 \mathrm{M}$ of NBD and to $2.5 \times 10^{-4}$ $\mathrm{M}$ of $\left[\mathrm{Ru}(\mathrm{bpy})_{3}\right] \mathrm{Cl}_{2}$.

2.3.2.2. Inkjet printing and graft copolymerization. Acrylic acid aqueous solutions were printed onto cellulose substrates using the Dimatix inkjet printer. Nozzle diameter was $21.5 \mu \mathrm{m}$ and nominal drop volume was $10 \mathrm{pL}$. Printing tests were performed at $30 \mathrm{~V}$ voltage with $15 \mu \mathrm{m}$ drop spacing. The printed pattern (Fig. 1) consisted of two solid forms of $1 \mathrm{~cm} \times 1 \mathrm{~cm}$ and $1 \mathrm{~cm} \times 0.2 \mathrm{~cm}$ dimensions. The pattern resolution was equal de 1693 dpi (dot per inch). Printings made of 1, 3 and 6 layers were compared. The patterned surfaces were irradiated at $453 \mathrm{~nm}$ $\left(0.75 \mathrm{~W} \mathrm{~cm}^{-2}\right.$ ) during 15 minutes (about $675 \mathrm{~J} \mathrm{~cm}^{-2}$ ) for inducing polymerization. After irradiation, printed substrates were dipped in distilled water during 5 hours to remove the physisorbed matter. They were finally dried for 60 minutes at $45{ }^{\circ} \mathrm{C}$ in an air oven.

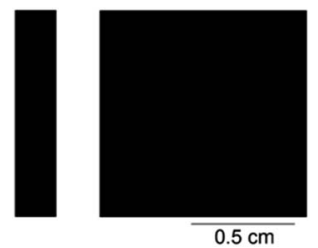

Fig. 1 Scheme of the printed pattern.

\section{Results and discussion}

\subsection{One-step cellulose graft copolymerization}

3.1.1. Molecular level. Whatman CF1 paper was selected because it is a high quality paper, made of quite pure and clean cellulose (Fig. 2), whose thickness and wicking properties are rather uniform (11 $\mu \mathrm{m}$ surface pore size and $176 \mu \mathrm{m}$ thick). Cellulose is a natural biopolymer made up of glucose units (Fig. 2). It is the simplest polysaccharide since it is composed of a unique monomer (glucose) which binds to its neighbors by a unique type of linkage ( $\beta-1,4$ glycosidic bond resulting in acetal function). ${ }^{1}$ Hydroxyl groups in glucose units are responsible for cellulose chemical activity. ${ }^{2}$ Among the three hydroxyl groups in each glucose residue, the hydroxyl at 6-position (primary one) is the most reactive site., ${ }^{\mathbf{1 , 2}}$

Cellulose paper sheets have been copolymerized in soft conditions, in a single step and after only one hour incubation. Several acrylic polymers were grafted. The molecular structures of the corresponding monomers are shown in Fig. 3. The graft

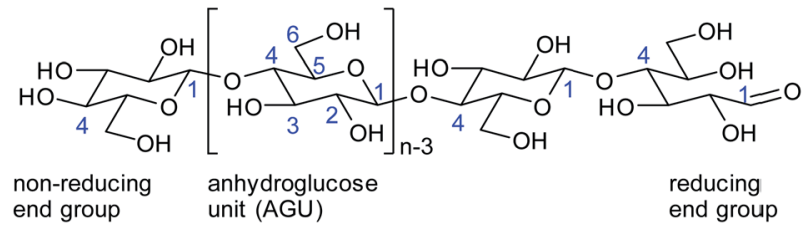

Fig. 2 Cellulose molecular structure.

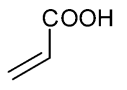

AA

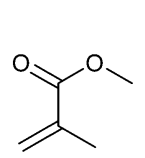

MMA

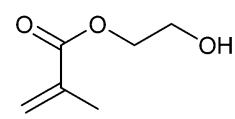

HEMA

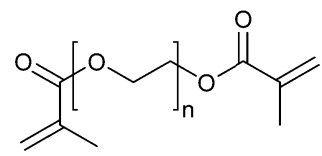

PEGDMA
Fig. 3 Molecular formulae of the monomers.

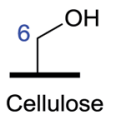

I

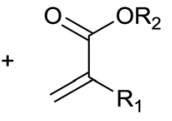

II

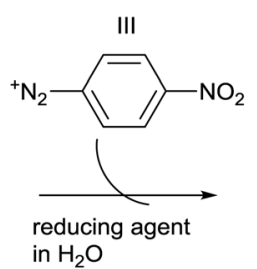

in $\mathrm{H}_{2} \mathrm{O}$
$\mathrm{R}_{1}=\mathrm{H}$ or $\mathrm{CH}_{3}$

$\mathrm{R}_{2}=\mathrm{H}$ or $\mathrm{CH}_{3}$ or $\mathrm{CH}_{2} \mathrm{CH}_{2} \mathrm{OH}$ or $\left(\mathrm{CH}_{2} \mathrm{CH}_{2} \mathrm{O}\right) \mathrm{nC}(\mathrm{O}) \mathrm{C}\left(\mathrm{CH}_{2}\right) \mathrm{CH}_{3}$

Fig. 4 Cellulose graft copolymerization with acrylic monomers. Aryldiazonium(III) is reduced and reacts with cellulose (I) in an aqueous medium to initiate the grafting and polymerization of the monomer (II) and give a polymer-grafted cellulose membrane (IV). 
polymerization pathway consisted in an aryldiazonium-based surface chemistry (Fig. 4). ${ }^{24,26}$ Diazonium salts are known to be free radical polymerization initiators. ${ }^{24}$ Acrylic graft copolymerization to the pre-existing polymeric cellulose backbone was therefore achieved by free radical graft copolymerization which was triggered by a reducing agent. According to the previously published work by Garcia et al. ${ }^{28}$ both "grafting-from" and "grafting-to" polymerization pathway are actually involved in the polymer grafting process. In light of the review of Roy et al. and works reported on it, ${ }^{2}$ we suggest the hypothesized mechanism depicted in Fig. 5. Reaction took place in water at room temperature with a biological reducing agent (L-ascorbic acid, also known as vitamin $\mathrm{C}$ ), thereby resulting in a biocompatible process. Cellulose sheets have been successfully grafted with the different polymers and characterized by several analytical techniques in order to assess the resulting surface chemical composition and morphological structure.

3.1.2. Surface chemical analysis. The outer surface layers of paper substrates were chemically analyzed by ATR-FT-IR, thereby displaying the aforementioned bulk molecular structures. According to its layout, ATR-FT-IR allows the identification of chemical bonds within $2 \mu \mathrm{m}$ deep subsurface layers. ${ }^{29}$ All papers are mainly composed of a cellulosic backbone and therefore the IR signals for its typical bond vibrations are shared by every spectrum shown. Fig. 6 displays these common bands attributable to $\mathrm{O}-\mathrm{H}, \mathrm{C}-\mathrm{H}, \mathrm{C}-\mathrm{C}, \mathrm{C}-\mathrm{O}$ and $\mathrm{O}-\mathrm{C}-\mathrm{O}$ stretching vibrations. As expected, polymer-grafted cellulose papers manifest additional peaks $\left(1725 \pm 5 \mathrm{~cm}^{-1}\right)$ attributable to $\mathrm{C}=\mathrm{O}$ stretching vibrations from ester moieties of the grafted polymer. Their intensity depends on the monomer used and resulting grafted polymer. They stand in the following order: AA $<$ HEMA

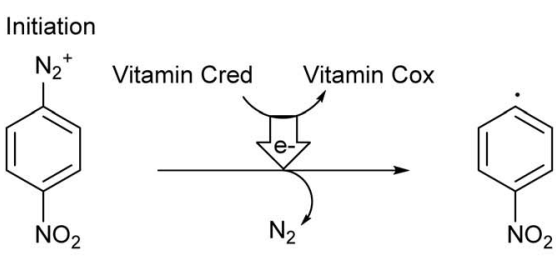

\section{Propagation}

Polynitrophenylene layer growth

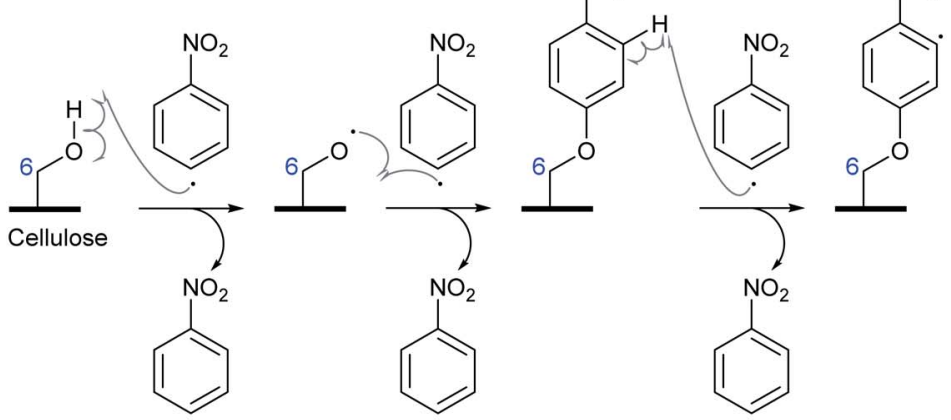<smiles>CC(C)Oc1ccc([N+](=O)[O-])c(-c2cccc([N+](=O)[O-])c2)c1</smiles>

"Grafting-from" pathway

Oligomer growth

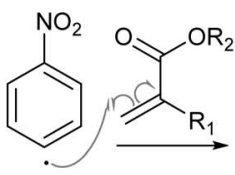<smiles>[R20]C(=O)C([R7])Cc1ccc([N+](=O)[O-])cc1</smiles><smiles>[R10]C(=C)C(=O)OCC</smiles><smiles>[R20]C(=O)CC([R7])(Cc1ccc([N+](=O)[O-])cc1)C(=O)OC([R2])[R]</smiles>

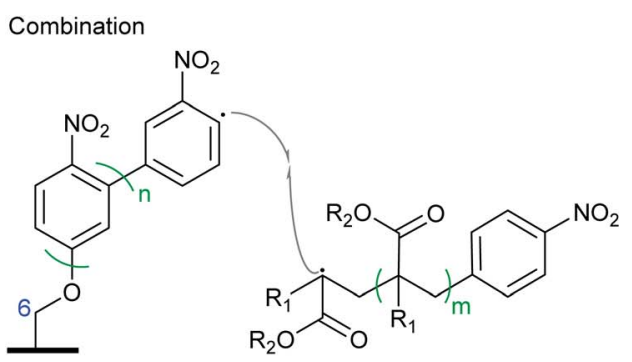

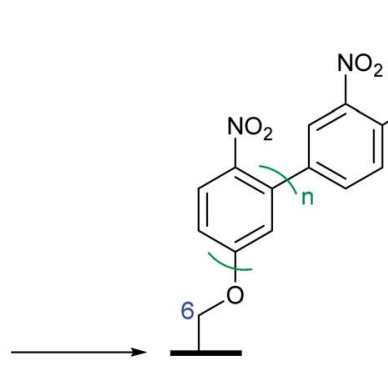

$\mathrm{R}_{2} \mathrm{O}$

"Grafting-to" pathway 


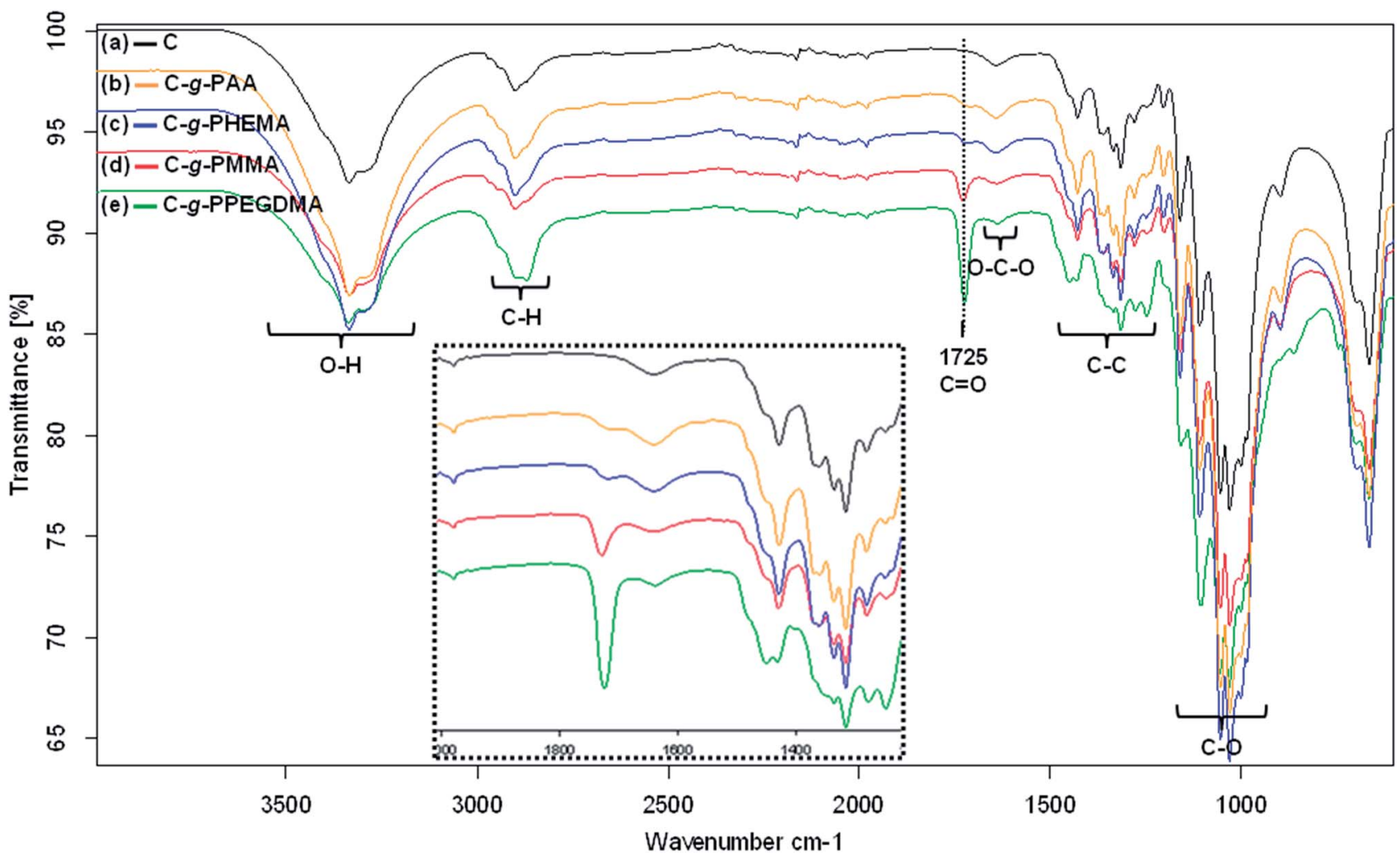

Fig. 6 IR spectra of paper substrates after a 1 hour dipping. (a) is spectrum from pristine cellulose sheet, (b) from cellulose copolymerized with acrylic acid (AA) monomer, (c) with 2-hydroxyethyl methacrylate (HEMA), (d) with methyl methacrylate (MMA) and (e) with poly(ethylene glycol) dimethacrylate (PEGDMA). All spectra have several bands in common which correspond to $\mathrm{O}-\mathrm{H}, \mathrm{C}-\mathrm{H}, \mathrm{C}-\mathrm{C}, \mathrm{C}-\mathrm{O}$ and $\mathrm{O}-\mathrm{C}-\mathrm{O}$ stretching vibrations. The $\mathrm{C}=\mathrm{O}$ stretching vibrations specific to grafted polymers are labeled.

$<$ MMA < PEGDMA. On one hand, PEGDMA is predominant because it is a diacrylic monomer. On another hand, since cellulose is a porous material these intensities cannot be directly related to amount and thickness of grafted polymer.
More investigations should be conducted in order to analyze surface morphological structure.

3.1.3. Surface morphological structure. Beyond the chemical differences in molecular structure, the various grafted polymers introduced physical and morphological differences

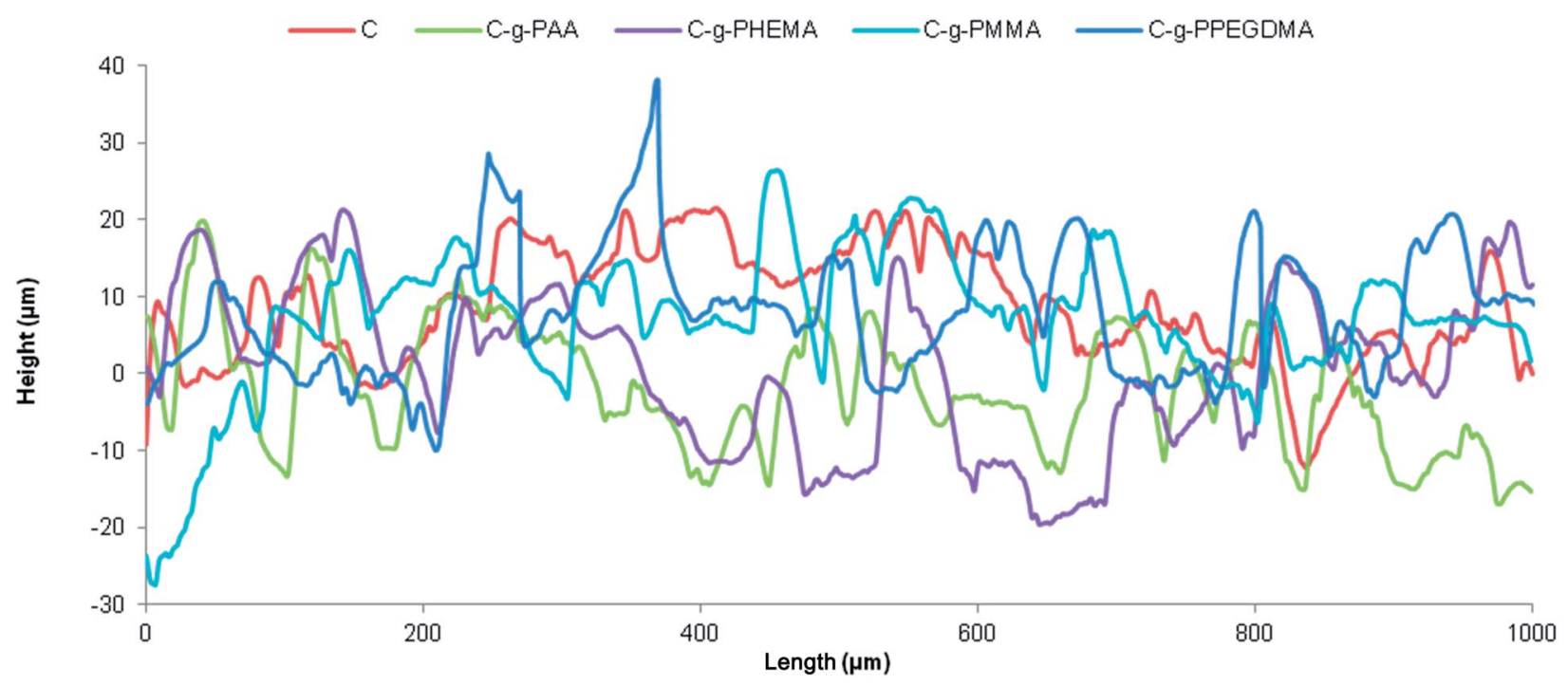

Fig. 7 Line profiles of pristine cellulose (C) and polymer-grafted cellulose substrates (C-g-polymer). 

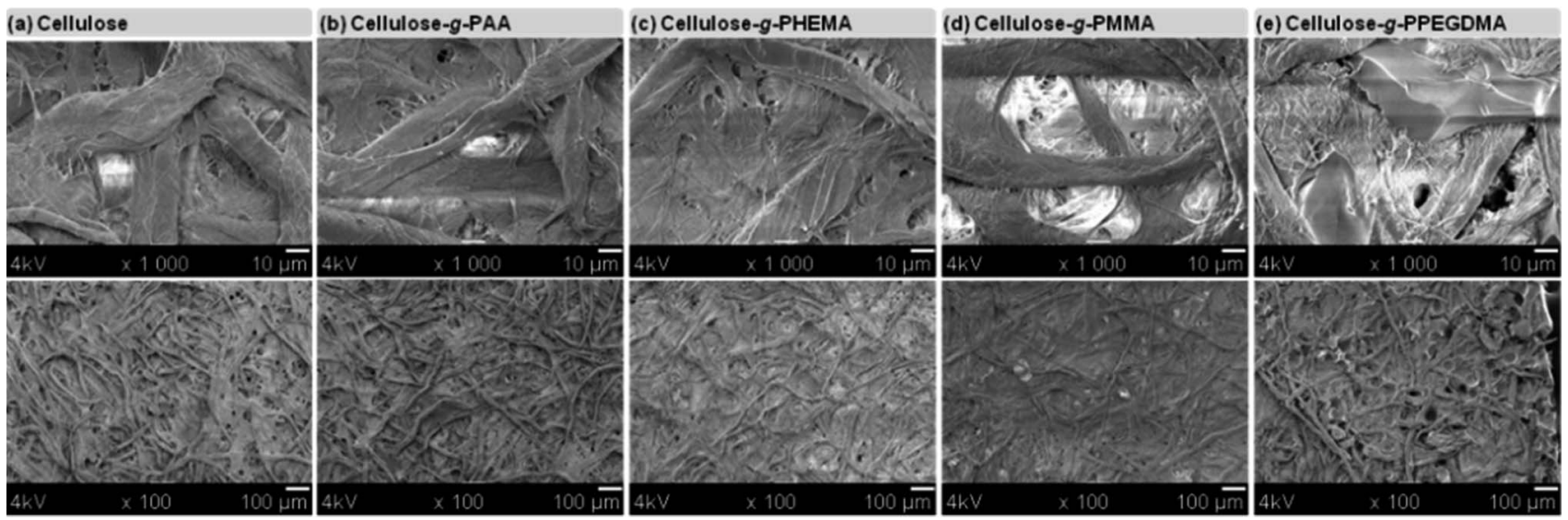

Fig. 8 Surface SEM micrographs of (a) pristine cellulose sheet, (b) cellulose copolymerized with acrylic acid (AA), (c) with 2-hydroxyethyl methacrylate (HEMA), (d) with methyl methacrylate (MMA) and (e) with poly(ethylene glycol) dimethacrylate (PEGDMA).

between the cellulose substrates. Thus, in order to quantify the variation in surface morphological structure, topological analysis was conducted by measuring substrates' roughness $\left(R_{\mathrm{a}}\right)$. Morphology and microstructure of the various polymer-grafted cellulose substrates was visualized by SEM imaging. Visual global evaluation was also performed.

Line profiles of pristine cellulose substrate and polymergrafted cellulose substrates were quite similar (see Fig. 7). Substrates were rather heterogeneous, rough, and displays numerous and wide pores. Surface roughness $\left(R_{\mathrm{a}}\right)$ values confirmed this high roughness and were also quite similar. Hence, the average value was $6.45 \pm 0.25 \mu \mathrm{m}$.

Pores sizes and arrangement pictured by SEM imaging (Fig. 8) were consistent with the previous statements. Pristine cellulose substrate and polymer-grafted cellulose substrates looked quite similar. They displayed numerous and wide surface pores. However, differences could be noticed between the various polymer-grafted cellulose substrates. Micrographs revealed that the grafted polymers filled cellulose surface pores, as expected. Progression of the filling extent matched the progression previously observed with IR peak intensities: AA < HEMA < MMA < PEGDMA. Therefore, these intensities could actually be related to an amount of grafted polymer.

Although the microstructures of the several cellulose substrates were different, the various substrates visually appeared quite similar. Except for cellulose-g-PPEGDMA which is slightly colored, grafted cellulose substrates were white and displayed no visual difference with pristine cellulose (Fig. 9). Cellulose molecular, physical and micro-morphological properties can therefore be modified without impact on the visual aspect of paper.

\subsection{Spatially controlled cellulose graft copolymerization}

Though the aforementioned process was ecologically friendly, the dipping procedure implemented was not economically friendly. Indeed, a large part of the reaction mixture was not involved in the cellulose graft copolymerization but in the homopolymerization of the added monomer. In order to reduce

this matter wastage, the polymerization was further localized onto selected specific areas of the substrate by means of inkjet printing. Printing is a versatile technique allowing the deposition of variable kinds of solutions (biomolecules, polymers, solvents, metals) onto different types of substrates (cellulose, polymer, glass, silicon) and according to any design desired..$^{30,31}$ This is fast dispensing process enabling low-cost, high throughput fabrication. ${ }^{31}$ Moreover it is regarded as an

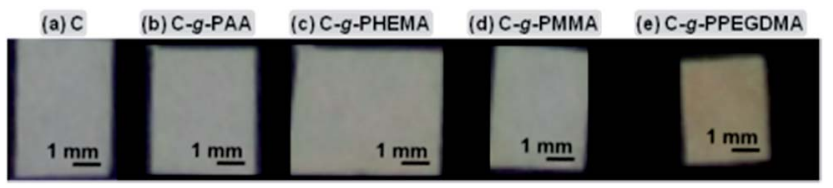

Fig. 9 Photographs of (a) pristine cellulose sheet, (b) cellulose copolymerized with acrylic acid (AA), (c) with 2-hydroxyethyl methacrylate (HEMA), (d) with methyl methacrylate (MMA) and (e) with poly(ethylene glycol) dimethacrylate (PEGDMA).

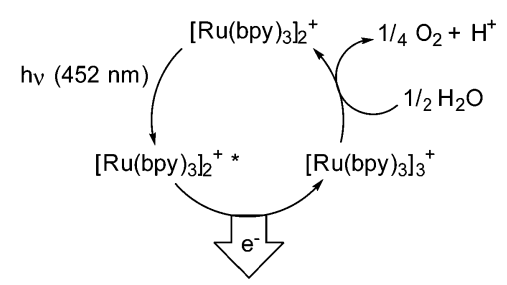

Fig. 10 Photoactivated reducing behavior of $\left[\mathrm{Ru}(\mathrm{bpy})_{3}\right]^{2+}$.

(a)

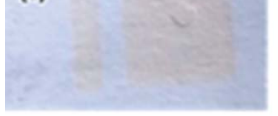

Fig. 11 Photographs of printed solid forms on cellulose substrates after irradiation and rising steps: (a) 1-pass printing, (b) 3-pass printing and (c) 6-pass printing. 
(a)

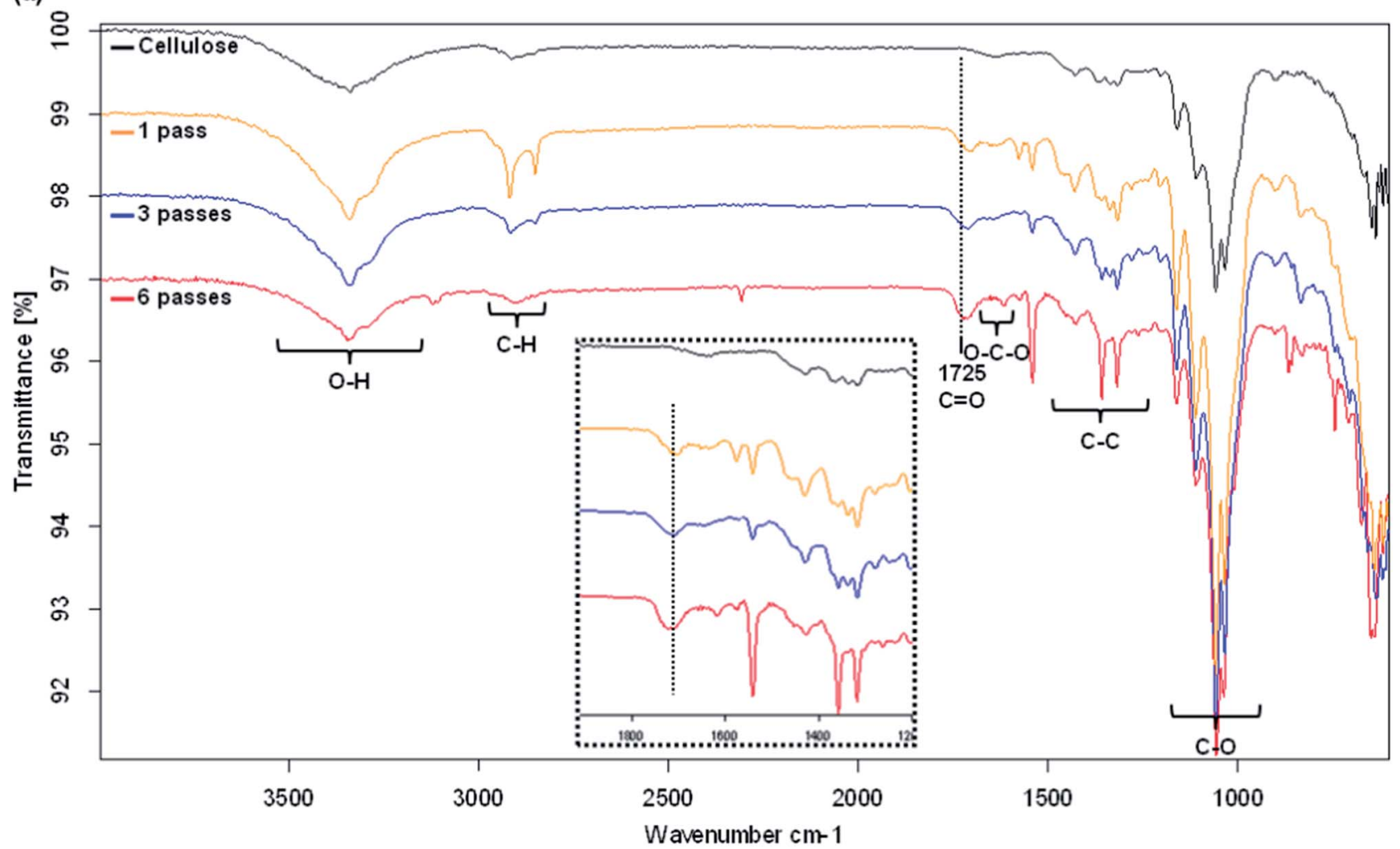

(b)

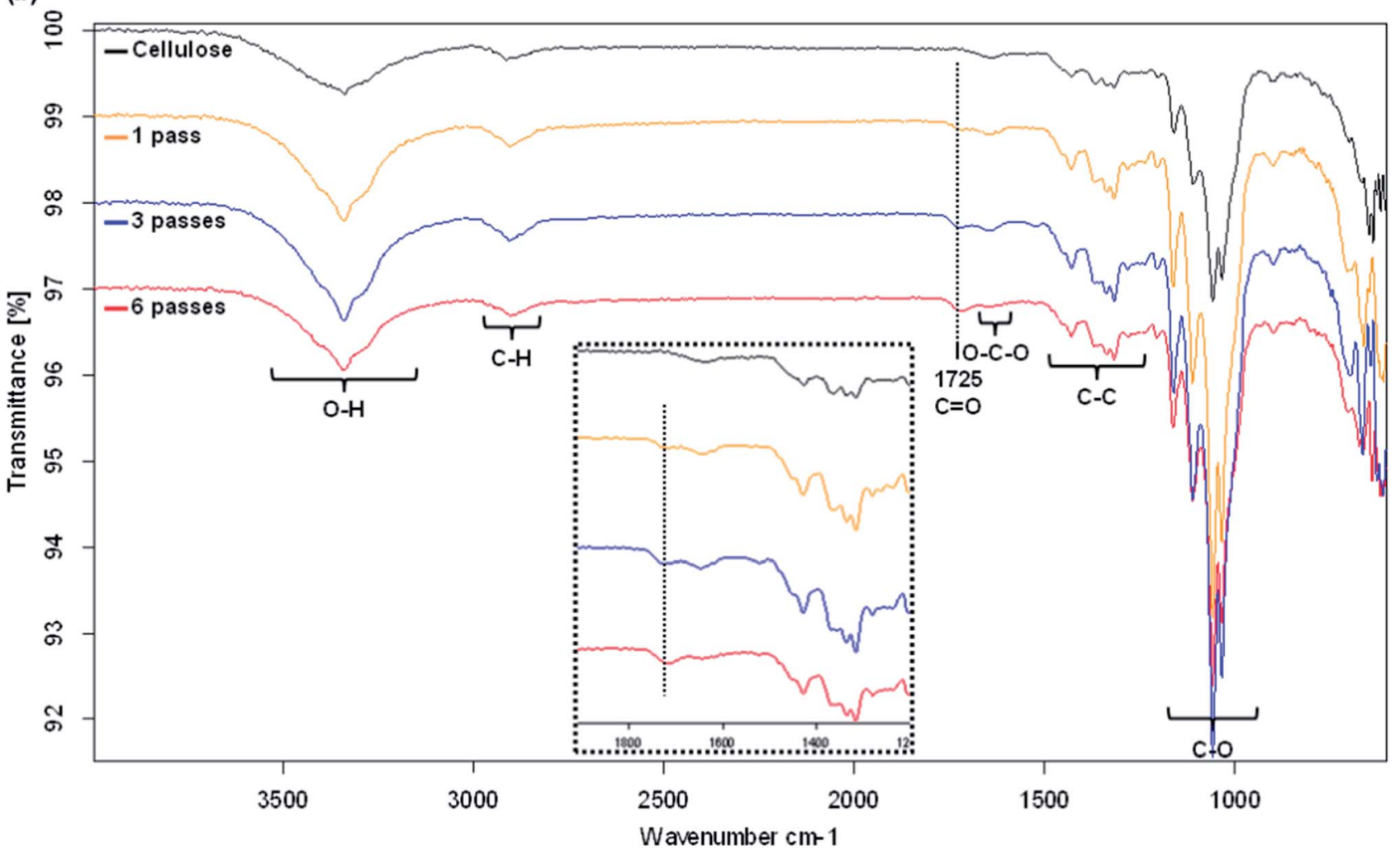

Fig. 12 IR spectra of raw cellulose and cellulose printed and copolymerized with acrylic acid monomer. 1-pass printing, 3-pass printing and 6 -pass printing were displayed. Spectra from the first set (a) were recorded before rinsing and those from the second (b) after rinsing. 
Table 1 Raw cellulose substrate roughness and printed polyacrylic acid films roughness and thickness

\begin{tabular}{lcccc}
\hline & \multicolumn{2}{c}{ Roughness $(\mu \mathrm{m})$} & & \multicolumn{2}{c}{ Thickness $(\mu \mathrm{m})$} \\
\cline { 2 - 3 } & Before rinsing & After rinsing & & Before rinsing \\
\hline Cellulose & $7 \pm 1$ & & & \\
1-Pass printed cellulose & $9 \pm 1$ & $7 \pm 1$ & $9 \pm 3$ & \\
3-Pass printed cellulose & $9 \pm 1$ & $8 \pm 2$ & $15 \pm 4$ & $4 \pm 2$ \\
6-Pass printed cellulose & $14 \pm 3$ & $9 \pm 1$ & $29 \pm 9$ & $13 \pm 8$
\end{tabular}

environmentally friendly process and therefore a very attractive approach regarding the economic and ecological goals.

However, the previous reaction mixture was not printable as was. The polymerization trigger had to stay inactive as long as it was in cartridge otherwise homopolymerization would have taken place before printing. Thus, vitamin $\mathrm{C}$ was exchanged for a photoactivated reducing agent: $\left[\mathrm{Ru}(\mathrm{bpy})_{3}\right]^{2+}$. $\left[\mathrm{Ru}(\mathrm{bpy})_{3}\right]^{2+}$ strongly absorbs at $452 \pm 3 \mathrm{~nm}$ in aqueous medium. ${ }^{32}$ In presence of oxidative quenchers such as aryldiazonium salt, the excited $\left[\mathrm{Ru}(\mathrm{bpy})_{3}\right]^{2+} *$ relaxed to $\left[\mathrm{Ru}(\mathrm{bpy})_{3}\right]^{3+}$ while transferring an electron to the aryldiazonium, thereby triggering cellulose graft copolymerization. $\left[\mathrm{Ru}(\mathrm{bpy})_{3}\right]^{3+}$ is a powerful oxidant $(1.29 \mathrm{~V} v s . \mathrm{SCE}=$ Standard Calomel Electrode, in $\mathrm{CH}_{3} \mathrm{CN}$ ) and would therefore be able to spontaneously oxidize water and return to its original $\left[\mathrm{Ru}(\text { bpy })_{3}\right]^{2+}$ form (Fig. 10). ${ }^{33-35}$

Reaction was still performed in water at room temperature. Cellulose sheets were successfully printed with a photoactive ink containing acrylic acid. Cellulose was further copolymerized by irradiating the printed pattern. Resulting substrates were characterized by several analytical techniques in order to assess their surface chemical composition and morphological structure.

3.2.1. Ink behavior. Before printing, ink rheological behavior was analyzed in order to check the printability of the prepared solution. To be inkjet printable, a fluid should be Newtonian with a viscosity in the range of 1 to $10 \mathrm{mPa} \mathrm{s} .{ }^{36}$ The formulated ink showed a Newtonian behavior with a constant viscosity of $3.2 \mathrm{mPa}$ s at shear rates varying from 100 to $5000 \mathrm{~s}^{-1}$. Shear stress varied linearly, from 0.3 to $16.2 \mathrm{~Pa}$, as a function of shear rate $\left(100\right.$ to $\left.5000 \mathrm{~s}^{-1}\right)$. The temperature was maintained at $24{ }^{\circ} \mathrm{C}$ during the whole measurements.
Then, inkjet printing was performed onto cellulose substrates. 1, 3 and 6 printing passes of acrylic acid aqueous ink were printed onto cellulose according to the pattern displayed in Fig. 1. Patterned surfaces were further irradiated, rinsed and dried. Photographs of the resulting printed forms are shown Fig. 11. When only 1 (Fig. 11a) and 3 (Fig. 11b) printing passes are deposited, both solid forms are well defined. However, when 6 printing passes are performed (Fig. 11c), the larger solid form is not homogeneous. This is probably due to the high ejected ink volume compared to the absorption capability of cellulose fibers. ${ }^{37}$

3.2.2. Surface chemical analysis. Fig. 12 shows IR spectra of raw cellulose and cellulose printed and copolymerized with acrylic acid. New peaks appeared on cellulose substrates at 1350,1530 and $1710-1730 \mathrm{~cm}^{-1}$ after light induced polymerization of acrylic acid. Peaks around 1530 and $1350 \mathrm{~cm}^{-1}$ are attributable to stretching vibrations of nitrophenyl groups from NBD derivatives. Peaks around $1710-1730 \mathrm{~cm}^{-1}$ are related to the stretching vibrations of carboxylic groups $(\mathrm{COOH})$. Peaks intensity was proportional to the number of passes. After rinsing in distilled water during 5 hours, peaks attributed to NBD could not be identified anymore. Furthermore, even though the carboxylic peaks $\left(1728 \mathrm{~cm}^{-1}\right)$ were still easy to discern, their intensity had decreased. This phenomenon is partially caused by the $\mathrm{COOH} / \mathrm{COO}^{-}$equilibrium resulting from the sustained exposure to distilled water. No copolymer's peak was observed anymore on the spectra corresponding to 1-pass printing. This could be explained by an ejected ink volume too small to allow surface polymerization of acrylic acid.

3.2.3. Surface morphological structure. Printing and graft copolymerization of cellulose with acrylic acid monomer resulted in the formation of a thin film of polyacrylic acid onto

\section{(a) Cellulose}

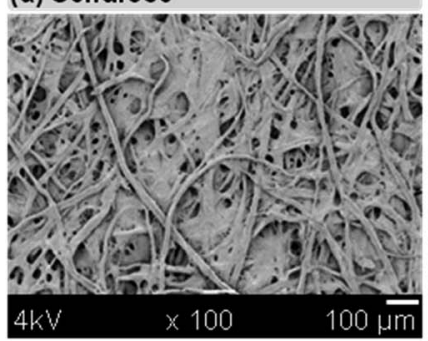

(b) Cellulose-g-PAA (1 pass)

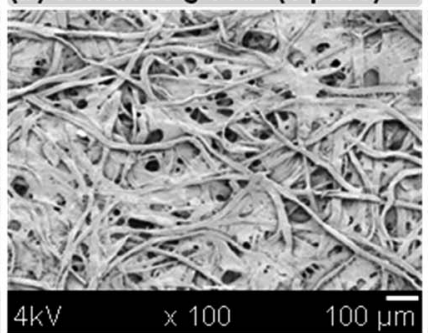

(c) Cellulose-g-PAA ( 3 passes)

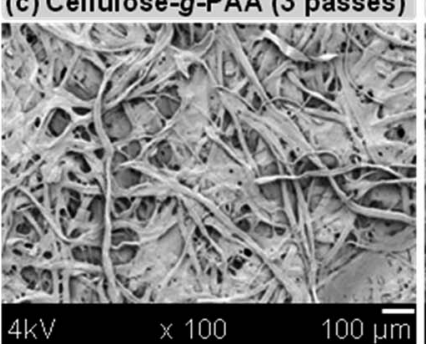

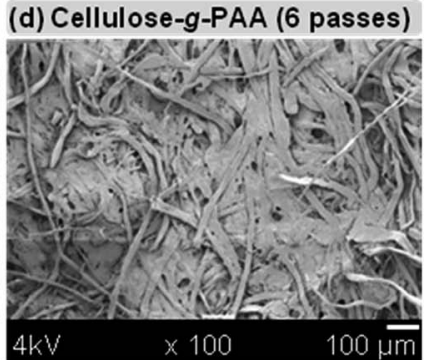

Fig. 13 Surface SEM micrographs of raw cellulose (a) and cellulose printed and copolymerized after rising step: 1-pass printing (b), 3-pass printing (c) and 6-pass printing (d) are displayed. 
(a) Cellulose-g-PAA (1 pass)

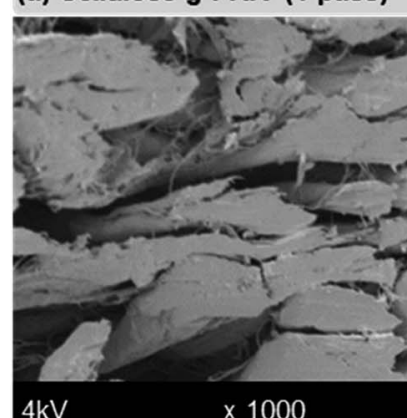

(b) Cellulose-g-PAA (3 passes)

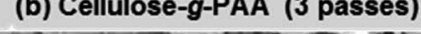

Fig. 14 Cross-section SEM micrographs of printed and copolymerized cellulose after rising step: 1-pass printing (a), 3-pass printing (b) and 6pass printing (c) are displayed.

cellulose sheet surface. Those films' thicknesses and roughnesses are displayed in Table 1. Roughness values were compared to raw cellulose one. Thus, printing and graft copolymerization made roughness increase from $7 \mu \mathrm{m}$ to $14 \mu \mathrm{m}$ (6pass printing). Grafted films thicknesses varied from $9 \mu \mathrm{m}$ for 1pass printing to $29 \mu \mathrm{m}$ for 6-pass printing. Washing printed substrates with distilled water further allowed removing the physisorbed material, thereby inducing thickness and roughness decrease. Besides, the film resulting from 1-pass printing completely vanished after rinsing. In this case, film thickness could not be measured and the film roughness was equal to raw cellulose one. This might stem from the complete absorption of the small ejected volume of ink by cellulose fibers and pores, hence inhibiting light induced polymerization of acrylic acid onto cellulose surface. Indeed, the grafting efficiency depends, inter alia, on the photoinitiator and the monomer concentrations. ${ }^{38}$ Thus, the ejected volume could be a determining factor outlining the thickness of the grafted polymer as well as the polymerization efficiency.

In order to further investigate films morphology, scanning electron microscopy was performed. Surface micrographs of raw cellulose and printed plus copolymerized cellulose are shown in Fig. 13. Firstly, raw cellulose and 1-pass printed cellulose looked almost identical. One may suggest that most of the ejected ink had been absorbed by the cellulose surface pores. Then, 3-pass printed and 6-pass printed cellulose appeared quite different from raw cellulose. Fewer pores are observed on the surface which seems more homogeneous, mainly after the 6-pass printing. This results are consistent with Määttänen et al. findings $\mathrm{s}^{39}$ which demonstrated that ink is quickly and completely absorbed into the depth of porous surfaces. Thus, in order to enhance ink deposit onto the substrate surface more passes should be performed.

Cross-section micrographs of printed and copolymerized cellulose are shown in Fig. 14. According to these micrographs, structure changed as a function of the passes numbers. Fewer pores were observed when the number of printings increased from 1 to 6 passes. Furthermore, cellulose sheet depth appeared more packed with the increasing number of passes. This confirmed the fact that ink penetrated through the cellulose capillarity and pores.

\subsection{Structural characterization}

XRD analysis of the PAA printed and dipped cellulose was performed in order to investigate the behavior of cellulose crystallinity with the different grafting processes (Fig. 15). Compared to untreated cellulose, the printing and dipping processes do not alter the structure of cellulose fibers.

\subsection{Inkjet printing of complex patterns}

As previously mentioned, one major advantage of inkjet printing dispensing method is the freedom in design of the printed pattern. This advantage was illustrated here by printing a copolymerization mixture according to the nature of the monomer and the resulting polymer grafted to cellulose. Therefore, acrylic acid aqueous ink was printed (6-pass printing) onto cellulose according to a pattern drawing the abbreviation PAA (Fig. 16a). Patterned surface was further irradiated, rinsed and dried. Photograph of the resulting printed form is shown in Fig. 16b. Visual aspect was consistent with previous results for a 6-pass printing (see Section 3.2.1). As expected, the drawn pattern allowed direct reading of the grafted polymer. Afterwards, a smaller and thinner pattern was printed in order to assess the resolution of the actually grafted film. Pattern and photograph are shown in Fig. 16a and b, respectively. They confirmed that this process enables to precisely modulate properties of a cellulose surface according to

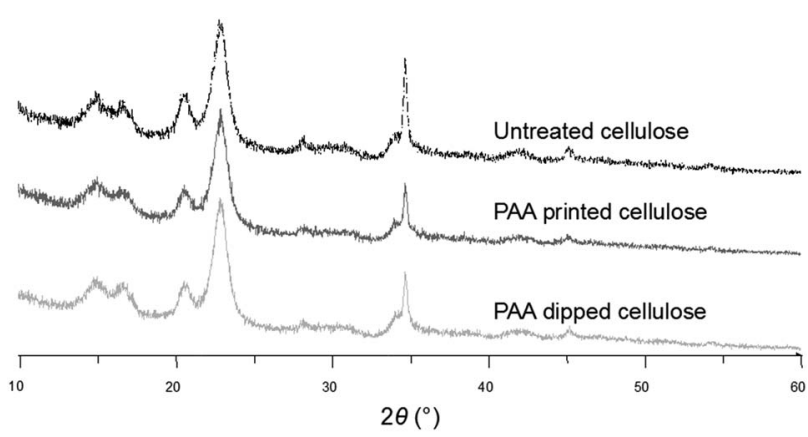

Fig. $15 \mathrm{X}$-ray diffraction spectra of untreated, PAA printed and PAA dipped cellulose. The XRD Data were collected using CuK $\alpha$ radiation. 
(a)

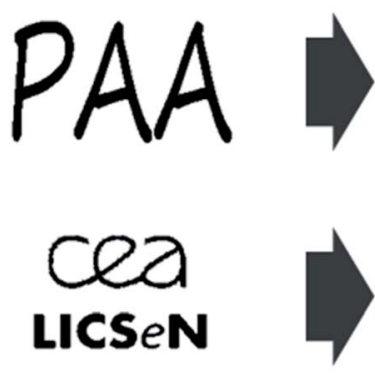

(b)
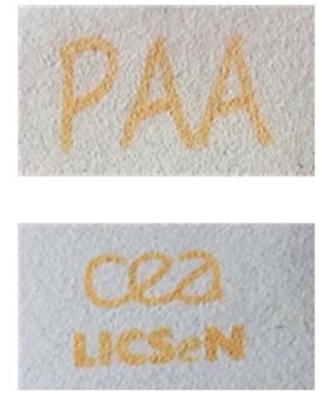

Fig. 16 Printed pattern designs (a) and photographs of the solid forms actually printed on cellulose (b).

complex patterns. Spatial control of surface properties is key asset of such a modification process. For instance, precise spatial control of electrical properties is particularly interesting in order to produce paper-based electronic circuit.

\section{Conclusion}

The work described herein offers a simple, fast, low-cost and eco-friendly way for cellulose surface graft copolymerization. This original approach, based on aryldiazonium salt chemistry, is achieved under soft aqueous conditions and through a onestep reaction. Cellulose sheets have been impregnated with copolymerization reaction mixture by means of two different dispensing methods. Firstly, dipping was performed and enabled to use a biological reducing agent: vitamin $\mathrm{C}$. The process was thus ecologically friendly but not economically friendly. This is why inkjet printing was further implemented. This versatile and economically friendly dispensing method ensured reduction of the matter wastage by localizing the polymerization mixture onto specific areas of the substrate. However, this process modification required to exchange vitamin $\mathrm{C}$ for a photoactivated reducing agent: $\left[\mathrm{Ru}(\mathrm{bpy})_{3}\right]^{2+}$. Several acrylic polymers were grafted to cellulose. Results suggest that the chemical pathway followed here allows graft copolymerization of cellulose sheet with many different acrylic monomers.

This research was proposed to meet the need of paperbased technology for cost and time-saving methods allowing robust and sustainable graft copolymerization of cellulose sheets. In addition to the simplicity of a one-step reaction, inkjet printing dispensing of the reaction mixture allows to precisely localize the polymerization and to save expensive monomers. Therefore, the expounded process provides a powerful tool for easy and robust graft copolymerization of cellulose sheets with various polymer films and according to complex patterns.

\section{Acknowledgements}

This work was financially supported by the Commissariat à l'Energie Atomique et aux Energies Alternatives (France).

\section{Notes and references}

1 J. Credou and T. Berthelot, J. Mater. Chem. B, 2014, 2, 47674788.

2 D. Roy, M. Semsarilar, J. T. Guthrie and S. Perrier, Chem. Soc. Rev., 2009, 38, 2046-2064.

3 R. L. Crawford, Lignin biodegradation and transformation, John Wiley \& Sons Inc, New York, NY, USA, 1981.

4 E. Malmström and A. Carlmark, Polym. Chem., 2012, 3, 17021713.

$5 \mathrm{~J}$. S. Han and J. S. Rowell, in Paper and composites from agrobased resources, ed. R. M. Rowell, R. A. Young and J. K. Rowell, CRC Press, 1996, pp. 83-134.

6 D. Klemm, B. Heublein, H.-P. Fink and A. Bohn, Angew. Chem., Int. Ed., 2005, 44, 3358-3393.

7 S. Kalia, B. S. Kaith and I. Kaur, Cellulose Fibers: Bio- and Nano-Polymer Composites, Springer, Berlin, Heidelberg, 2011.

8 D. Klemm, F. Kramer, S. Moritz, T. Lindström, M. Ankerfors, D. Gray and A. Dorris, Angew. Chem., Int. Ed., 2011, 50, 54385466.

9 R. Pelton, Trends Anal. Chem., 2009, 28, 925-942.

10 A. W. Martinez, S. T. Phillips, G. M. Whitesides and E. Carrilho, Anal. Chem., 2010, 82, 3-10.

11 J. Credou, H. Volland, J. Dano and T. Berthelot, J. Mater. Chem. B, 2013, 1, 3277-3286.

12 A. W. Martinez, S. T. Phillips and G. M. Whitesides, Proc. Natl. Acad. Sci. U. S. A., 2008, 105, 19606-19611.

13 SENTINEL, Bioactive Paper Network, http://www.bioactivepaper. ca/index.php? module $=$ page $\& i d=4000$, accessed 31 January 2014.

14 S. Aikio, S. Grönqvist, L. Hakola, E. Hurme, S. Jussila, O.-V. Kaukoniemi, H. Kopola, M. Känsäkoski, M. Leinonen, S. Lippo, R. Mahlberg, S. Peltonen, P. Qvintus-Leino, T. Rajamäki, A.-C. Ritschkoff, M. Smolander, J. Vartiainen, L. Viikari and M. Vilkman, Bioactive paper and fibre products: Patent and literary survey, Oulu, Finland, 2006.

15 Materials Research Society, MRS Bull., 2013, 38, 294-352.

16 D. Klemm, B. Philipp, T. Heinze, U. Heinze and W. Wagenknecht, Comprehensive Cellulose Chemistry Volume 2 Functionalization of Cellulose, WILEY-VCH, Weinheim, 1998, vol. 2.

17 J.-F. Zhong, X.-S. Chai and S.-Y. Fu, Carbohydr. Polym., 2012, 87, 1869-1873.

18 E. Takács, L. Wojnárovits, É. Koczog Horváth, T. Fekete and J. Borsa, Radiat. Phys. Chem., 2012, 81, 1389-1392.

19 R. Bongiovanni, S. Marchi, E. Zeno, A. Pollicino and R. R. Thomas, Colloids Surf., A, 2013, 418, 52-59.

20 Y. Kodama, M. Barsbay and O. Güven, Radiat. Phys. Chem., 2014, 94, 98-104.

21 E. Takács, L. Wojnárovits, J. Borsa and I. Rácz, Radiat. Phys. Chem., 2010, 79, 467-470.

22 W. Dahou, D. Ghemati, A. Oudia and D. Aliouche, Biochem. Eng. J., 2010, 48, 187-194. 
23 K. Littunen, U. Hippi, L.-S. Johansson, M. Österberg, T. Tammelin, J. Laine and J. Seppälä, Carbohydr. Polym., 2011, 84, 1039-1047.

24 V. Mévellec, S. Roussel, L. Tessier, J. Chancolon, M. MayneL'Hermite, G. Deniau, P. Viel and S. Palacin, Chem. Mater., 2007, 19, 6323-6330.

25 T. Berthelot, A. Garcia, X. T. Le, J. El Morsli, P. Jégou, S. Palacin and P. Viel, Appl. Surf. Sci., 2011, 257, 3538-3546.

26 V. Mévellec, S. Roussel, S. Palacin, T. Berthelot, C. Baudin, A. Trenggono and G. Deniau, WO 2008/078052, 2006.

27 R. Faddoul, N. Reverdy-Bruas and A. Blayo, Mater. Sci. Eng., $B, 2012$, 177, 1053-1066.

28 A. Garcia, N. Hanifi, B. Jousselme, P. Jégou, S. Palacin, P. Viel and T. Berthelot, Adv. Funct. Mater., 2013, 23, 3668-3674.

29 PIKE Technologies, Product Data Sheet, MIRacle ATR, Madison, WI, USA, 2014.

30 Printed Organic and Molecular Electronics, ed. D. Gamota, P. Brazis, K. Kalyanasundaram and J. Zhang, Springer, Boston, MA, US, 2004.
31 J. Birkenshaw, Printed Electronics (Pira on printing), Leatherhead, Surrey, UK, 2004.

32 X. Zhou, D. Zhu, Y. Liao, W. Liu, H. Liu, Z. Ma and D. Xing, Nat. Protoc., 2014, 9, 1146-1159.

33 M. Hara, C. C. Waraksa, J. T. Lean, B. A. Lewis and T. E. Mallouk, J. Phys. Chem. A, 2000, 104, 5275-5280.

34 J. M. R. Narayanam and C. R. J. Stephenson, Chem. Soc. Rev., 2011, 40, 102-113.

35 F. Teplý, Collect. Czech. Chem. Commun., 2011, 76, 859-917. 36 R. Leach and R. Pierce, The Printing Ink Manual, Springer, Boston, MA, US, 1993.

$37 \mathrm{M}$. Bergh, Absorbent cellulose based fibers investigation of carboxylation and sulfonation of cellulose, Chalmers University of Technology, Göteborg, Sweden, 2011, pp. 1-57.

38 A. Bhattacharya and B. N. Misra, Prog. Polym. Sci., 2004, 29, 767-814.

39 A. Määttänen, P. Ihalainen, R. Bollström, M. Toivakka and J. Peltonen, Colloids Surf., A, 2010, 367, 76-84. 